\title{
A REVIEW ON PATIENT-CONTROLLED ANALGESIA INFUSION SYSTEM
}

\author{
USHA RANI SHOLA, NEELA NARAYANAN V
}

\author{
Department of SCSE, School of Computing Science and Engineering, Vellore Institute of Technology, Chennai, Tamil Nadu, India. Email: \\ asha.s@vit.ac.in
}

Received: 23 January 2017, Revised and Accepted: 03 March 2017

\begin{abstract}
PCA is a patient-controlled analgesia infusion pump, which is used to infuse the medicine into the patients after surgery. It contains a syringe with pain medicine to infuse that is prescribed by the physician. The drugs used for pain control are high-alert medicines, since overmedication may cause death to the patients. These types of unbearable events may happen due to medical errors, prescription errors, adverse events (AEs), etc. Hence, it requires a precautionary attention or continuous monitoring for PCA pump infusion patients. However, always physicians or nurses may not monitor a patient continuously. To provide safety to the patient, the PCA pump needs a smart care process to alert the physician. This study represents the survey on PCA pump errors, AEs, and solutions for it to avoid them. The solution will automatically alert the infusion-related situation of the patients, those are taking the intravenous drug infusion at different procedure rooms in the hospitals. Moreover, it increases the safety to infusion pump with advances of decision-making in health, patient monitoring, alert notification to nursing, and productivity. This quality care can be achieved by integrating the PCA pump with other intelligent systems.
\end{abstract}

Keywords: Bar-coded medication administration system, Computerized provider order entry, Electronic medication administration record, Interoperability, Patient-controlled analgesia.

(C) 2017 The Authors. Published by Innovare Academic Sciences Pvt Ltd. This is an open access article under the CC BY license (http://creativecommons. org/licenses/by/4. 0/) DOI: http://dx.doi.org/10.22159/ajpcr.2017.v10s1.19588

\section{INTRODUCTION}

A patient-controlled analgesia (PCA) is a computerized pump, contains a syringe for medication which is used to control the pain of patients after surgery or to obstetric, terminally ill, and trauma patients. This medication is prescribed by a doctor and is connected directly to patient's (intravenous [IV]) line. The prescribed medication is suggested by the physician or caretaker in small constant flows as a program into the pump. The programming feature of the pump allows the physician to select the drug concentration, how much rate the drug rate can be infused (bolus rate) and how many minutes the pump should be programmed. The drug concentration levels are specified in $\mathrm{mg}$ or $\mathrm{mL}$, and the concentration rate is specified in $\mathrm{mg} / \mathrm{hr}$ or $\mathrm{mg} / \mathrm{min}$ or $\mathrm{mcg} / \mathrm{hr}$ based on the continuity of the rate.

Additional [1] doses of medication can be self-administrated by the patient as needed (if patient feels more pain) by pressing a button that is attached to the pump. That is why, it is called as patient-controlled pump. However, patients cannot press the button of the pump if they are feeling too sleepy. During such cases, the caretaker may press the push button for excess medication to control patient's pain.

Most of the opioids used for PCA pump include morphine, hydromorphine, other analgesia such as citalopram with meperidine, and fentangl. Normally, these medicines are infused into the patients intravenously and these are high-risk medicines which cause harms to patients if the medication management was not happened properly. The harms include injuries and even death to the patients. Medication management is necessary when infusing these risky medicines through the patients intravenously.

\section{HISTORY OF INFUSION PUMPSS}

During 1990s [2] with the arrival of EEPROM, the infusion pumps became the smart pump with safety software. This safety software alerts the hospitals if the pump exceeds the limits. In 2000s, 10 years after the introduction of the smart pump, few of the developments are included. A dose error reduction system (DERS) is the one which prevents and identifies the errors in prescription and programming. Devices with these systems are commonly termed as "smart pumps." This system alerts the clinician when he/she tries to change the drug concentration against the predefined limits. The developments included here are dose rate limits, standardized drug concentration and its units, documentation of prevented programming errors, and identification of the manual errors. This experience suggested the inclusion of smart infusion pump with wireless connectivity/server applications. This is included into smart pump in 2003. Then, the pump has wireless connectivity and a server application. It is basically bidirectional connectivity between many infusion pumps and a single server. This allows the hospitals to get the infusion data and to revise the software uploads to the pump. It greatly improves the infusion pump safety and the continuous quality improvement efforts [2]. During the study of infusion pumps in 2010, the following limitations are noticed from 600 in process infusion pumps [2]:

- Assignment of specific pump to a particular patient: It is not ensured association of the drug, the amount of dose rate, and the time to a unique patient.

- No idea of the patient treatment

- No documentation of physician alerts history and infusion data

- Poor submission of DERS use or avoiding of alerts [2]

- No intimation of alarms, alerts, or infusions status.

To ensure all these requirements to the smart pump, many other systems need to be integrated to make it to behave as a more intelligent infusion pumping systems. This opens the door of interoperability to the smart pump system. This requires that many systems need to be integrated with the infusion pump. With that integration, the infusion pump can be named as intelligent infusion pump.

\section{EXISTING TECHNOLOGIES FOR GENERAL INFUSION PUMPS}

1. Smart pump including software program: Here, the infusion pump [8] is added with a customized drug library known as DERS. This library alerts users when predefined infusion min and max values are in abnormal conditions. The inclusion of this software [DERS library] to the infusion pump is termed as smart pump. However, it causes the administration errors.

2. Smart pump with barcode technology: The barcode technology is used to identify the patient and the drug. It assures the delivery of right drug to the right patient. It would be implemented with an added interface between the Hospital information systems (HIS), the pump along with barcode scanner for identification. This system 
needs the integration of servers system, bar-coded point of care (BPOC) system, and documentation process of HIS.

3. Integrated smart pump with large medication safety system: This system integrates the smart pump with barcode technology, computerized prescribed order entry (CPOE,) and electronic medication administration record. Two types of alerts can be included in the pump.

\section{Soft alerts}

Alerting the user about drug level and allows further administration of drug dosage levels.

\section{Hard alerts}

Alerting the user about drug level but does not allow the administration of drug dosage levels further.

The effect of different error of infusion pump is given in Table 1.

\section{Adverse events (AEs) of PCA pump}

An unintended or an unexpected outcome that harmfully affects a patient's health which may extent the treatment of the one required or everlasting damage of patient is termed as AE of PCA pump. Or any problematic medical incidence in a patient or clinical enquiry subject administered a medical product and which does not necessarily have to have a causal relationship with this therapy termed as [3] "AE." This may happen when delivering the medicine to the patient. Moreover, it causes not because of the medicine that was injecting. It may happen because of oversedation of the drug: Respiratory distress. These events occurs because of harms caused by the medial device or clinical management, but not because of the patient's illness. Some injuries results from medical intervention happens based on the preprogrammed medicine through Intravenous patient controlled analgesia pump (IV-PCA). Moreover, delivering the wrong medicine and wrong amount causes these events. The use of PCA pump entails more hazards [2] than the use of other types of pumps. As these PCA pumps use high potential drugs, even a small error can also lead to serious patient harm. The US FDA's manufacturer and user device experience (MAUDE) data base reveals that the PCA related device events are three times [2] higher risk than the events involving with the general infusion pumps. [Manufacturer and User Facility Device Experience] the medical device events with respect to PCA are categorized into five events namely,

- Patient-related events: $12(0.6 \%)$

- Device safety events: $1590(79.1 \%)$

- Operator errors: 131 (6.5\%)

- Adverse reactions to opioids: 25 (1.2\%)

- Indeterminate: 235 (11.7\%)

Even though few of the problems can be preventable (respiratory depression using threshold), adverse drug events (ADEs) of operator error will result most severe consequences than other events. The consequences include deaths, injuries, and respiratory arrest. The device safety events may not cause much effect on patients, but the operator error results over delivery of drugs which is very dangerous harm of PCA-related event. Most of the ADEs of PCA can be preventable but while using this pump for delivery carries a high risk of potential harm to the patients, a special attention of these ADE must be warranted.

\section{Opioids related AEs}

Possibility of respiratory depression during the use of opioids (highalert medicines) in hospitals is known as opioid-related AEs. It is like anoxic brain injury that causes due to the cardiopulmonary arrest. These events are termed as failure to saving. This is the first and third most common cause for AEs related to patient safety. Hence, warnings need to be alerted against the infusions of these opioids. In a clinical point of view, respiratory depression during drug injection to patient during post-operative period remains serious patient safety risk. From 2005-2009 MAUDE, 56000 AEs and 7000 patients deaths related to PCA.

\section{PROBLEMS WITH PCA INFUSION PUMP}

Based on the overall events with PCA, the problems can be categorized as:

1. PCA by proxy

2. Programming errors

3. Prescription errors

4. Not maintained the records of infusions, not maintaining the reasons for override alerts and caregiver information

5. Incorrect selection of drug mix-ups

6. Continuous monitoring of physiological parameter to alert overdosed high alert IV fluids

\section{PCA by proxy}

In PCA, the pumping of medicine can be controlled either by a patient (by pressing the PUSH button) [4] or a family member without having the knowledge and training on how to use the PCA pump. This leads to most tragic errors or AEs which are known as PCA by proxy errors. This error causes the patient oversedated from having actual received. The proxy (patient or caretaker or family member) will press the button of the PCA to manage his/her (patient's) pain. Because of this, sometimes the patient is receiving too much opiate or additional doses of the drug. Hence, it is necessary that clinicians should communicate that allowing anyone delivery request of PCA button other than the patient is a clear restriction of PCA therapy and has been strongly warned against by the Authority, ECRI Institute, ISMP, and the Joint Commission [2].

\section{Programming errors}

Delivering wrong medication or wrong concentration through PCA pump reports more serious hazards. These errors are inaccurately setting program parameters either low or high concentration of drugs to the infusion pump. For example consider a caregiver programs the dosage levels from $0.1 \mathrm{mg} / \mathrm{ml}$ of morphine rather than $1 \mathrm{mg} / \mathrm{ml}$., because of this the patient will receive 10 fold higher doses than the actual amount. Programming the pump as 84 instead of 8.4, 900 instead of 9 , or entering the volume as the rate could each deliver a fatal dose [2]. Such type of incorrect programming values causes the vital parameters changes of patient.

MAUDE database of 2011 reports nearly 4230 such errors. From that, $70 \%$ of reports are associated with misprogrammed doses and misuse of the pump. The concentration would be programmed as ordered but a higher concentration bag would be nominated and coupled to the patient through pump [2]

Table 1: Implementation of different levels of infusion pumps

\begin{tabular}{|c|c|c|c|}
\hline S. No. & Technology & Tested on patients & Reduce of errors \\
\hline 1 & Smart pumps with soft alerts & $\begin{array}{l}\text { ICU patients, cardiac surgery patients, and } \\
\text { pediatric patients }\end{array}$ & $\begin{array}{l}\text { Adverse events, medication errors, } \\
\text { infusion errors, and dose errors }\end{array}$ \\
\hline 2 & Smart pumps with soft and hard alerts & Common hospitals and academic medical center & Length of stay \\
\hline 3 & $\begin{array}{l}\text { Smart pump with soft, hard alerts, and barcode } \\
\text { technology }\end{array}$ & Simulation study & $\begin{array}{l}\text { Wrong drug errors, patient ID } \\
\text { errors, overdose errors }\end{array}$ \\
\hline 4 & $\begin{array}{l}\text { Smart pump with soft, hard alerts with } \\
\text { integrated barcode technology and EMAR }\end{array}$ & A program on intelligent infusion devices & Pump programming errors \\
\hline
\end{tabular}

EMAR: Electronic medication administration record 


\section{Example}

PCA was ordered for morphine $1 \mathrm{mg}$ dose, 8 minutes lock-out with $10 \mathrm{mg}$ hourly limit. PCA morphine concentration comes as $1 \mathrm{mg} / \mathrm{ml}$ standard. Moreover, the physicians intended the concentration rate as $2.5 \mathrm{mg}$; all the dose and lock-out time limits are set but the concentration rate was set to $0.25 \mathrm{mg}$ instead of $2.5 \mathrm{mg}$ and PCA pump was programmed as morphine. Because of this, the dose was giving 4 times [2] ordered dosage each time.

\section{Prescription errors}

Sometimes, the patients have been infused with the drug one that not prescribed leads to serious errors such as opioid toxicity (poisonousness). These errors includes with the following:

- Errors in selecting the opioids suitable to the patients

- Wrong drug dosage injection instead of the prescribed one

- Wrong understanding of the drug dosages

- Clinicians miscommunications.

The errors may happen when selecting the opioid that is not suitable to the patient. For example, on prescribing the meperidine for renal impairment, the patient has been ordered for different opioid.

\section{Example}

While in the prescription, if the dosage is written with $2.0 \mathrm{mg}$, the clinician understands it as $20 \mathrm{mg}$ and ordered for it; if it is intended, nearly 10 times higher dose than the required one will be received. This can be avoided by entering the prescription entry through the computer and the data need to be verified by the physicians once.

\section{Maintaining the records of infusions and record of caregiver}

Because of the above errors, the patient may be infused overdose or he/she may be poisoned. Moreover, the alerts that happen sometimes would be overridden by the nurse and that leads to a serious AE. One of the reasons behind these events is not maintaining the records for infusion order, drug order, and alerts. Hence, the doctor's prescription should be entered through computer order entry and the infusion order should be informed to the physician before the process to start. For example, in some scenarios, the prescribed medicine may be discontinued. As this information is not documented, the infusion will be continued unknowingly. This may lead to few of the physic parameters to reduce and the patients under critical conditions are not be recognized. For example, the low-level condition of potassium does not recognized by the nurse. Hence, he/she will not administrator the next dose to proceed for precaution. These systems can be overcome through clinical alerts from the intelligent systems. This will automatically flag the staff and then the staff can take appropriate actions to rescue the patient from harm.

An integrated wireless connectivity with the patient medical administration system and HIS will put some check to the above problems. Associating the pump with a particular patient is one of the main features to the interoperability of the infusion pump. The pump can be associated with a patient in any of the following ways;

- Entering the patient ID into pump through its keypad or

- Scanning the barcode of the patient ID from his/her wristband through barcode scanner integrated with the pump. Or

- Using the BCMA scanner application between the patient and the pump.

With this association of pump and patient, difference events of dosage errors (DERS events) can be analyzed easily and this history can be used later point of times to avoid the events that may occur in future. The brief discussion on BCMA is given in later sections.

\section{Incorrect selection of drug mix-ups}

Some harms are occurring when the drug mix-ups are selecting with other pairs of incorrect opioids. For example [2] two women while in labor receive incorrect opioids resulted overdoses of drugs and their babies developed respiratory difficulties. These errors are occurring due to the fact that few opioids used for PCA have common names and packaging. Thirty percent of the opioids look-alike the same name events reports [2]. This problem can be rectified with the pharmacy applied medicines labels against the prescription list of the patient.

Intensive monitoring (postoperative opioid-induced respiratory depression) and high alert overrides (continuous monitoring of physiological parameter to alert overdosed high alert IV fluids)

Patients receiving the opioids through PCA are at risk of respiratory depression or respiratory arrest due to the use of medications such as morphine, hydromorphine sedations. These are infusing through IV line to the patients and are high alert drugs. Moreover, alert is not identified easily when dosage levels are high. For example, a chemical such as morphine infused to post-operative patients for pain management may lead to respiratory depression if overdose of medication is taken by the patient. According to Anesthesiology, in 2010 considered postoperative respiratory failure is the $3^{\text {rd }}$ most common patient-related AE's affect. On account of this respiration depression AE, many of the hospitals are charged heavily.

Once Amanda, an 18-year-old female has been to the treatment in a hospital with a virus [2] and dehydration. She was admitted to hospital as it causes a great pain in her mouth and throat. She needs to put on with rehydration and antibiotics. On the next day, she was connected with PCA pump with morphine. The morphine [1] that the hospital staff giving her was not getting rid of the pain. Moreover, Amanda's tonsils and uvula were extremely swollen. She was not able to eat and drink anything. To manage her pain, Amanda was put on a PCA pump that allowed her to control the pain medication using hydromorphone. The next day morning she passed away due to respiration arrest. The reason behind her death was as not monitoring of physiological parameters continuously. If an electronic monitoring is connected with the PCA, the vital data of Amanda's will alert the nursing staff from the respiration arrest and she would have given other medication and she alive on today.

PCA pump basically provides [1] management of pain for patients. To provide the patient safety, patient should be connected with continuous electronic monitoring. This electronic monitor system provides the continuous measurement of two physiological parameters. Pulse oximetry measures the levels of oxygen in the blood stream, and capnography measures the carbon dioxide in the lungs are the two devices used for monitoring. This needs to be a standard practice for all PCA patients.

Pediatric patients, patients who are obese, with obstructive sleep apnea, and elder ones should be examined more carefully when these medications are infusing. The anesthesia patient safety foundation recommended that continuous electronic monitoring should be used for patients receiving the analgesics. During this process, the patient's basic physiological parameters such as respiration rate, patient's condition, and the supplemental of oxygen should be monitored. For example, the post-operative infusion respiration depression is one of the AEs that may occur during the infusions of PCA infusion pump. This depression may lead the patient to coma or death if not noticed earlier. Here, the pump alerts the cutoff levels of the physiological parameters when they are reaching beyond the threshold levels for example if no of breaths per minute $<10$.

\section{PROPOSED SOLUTION}

The intelligent PCA infusion safety system can able to identify medication error, patient monitoring errors, the drug order errors, the dosage levels of programming, identification of patient techniques and periodical checking of patient infusion levels and physiological parameters. To provide safe infusion pump, the following key points needs to be considered:

- $\quad$ The pump needs to be connected through wireless network

- And this pump should be associated with patient and a clinician

- Barcode and wireless frequency identification should be considered 
to recognize the IV drug/medication being infused

- Pump should be programmed automatically without the manual programming and is according to the physician's prescription order

- This pump should be protected from programming errors like drug infusion errors, over dosages, prescription errors, etc.,

- The history of infusion pump should be automatically recorded in every patient's EMR

- The patients getting the high alert IV medications should be monitored continuously for vital sign monitoring

- The pump signals and alarms should be delivered to the caregivers directly

- The pump maintenance issues should be sent to the biomedical staff automatically.

Fig. 1 represents the problems in smart infusion system and proposed solutions for the errors. And the proposed intelligent infusion system is represented in Fig. 2.

\section{Barcoded medication administration (BCMA)}

BCMA can help ensure the unit-dose solutions, and injections with the drug administration of the patient. This connects the patient and the pump. BCMA is a database system that has list of barcodes used for preventing the medication and human errors involved with prescription. It ensures that right medication is received by the right patient by electronically checking the documented medicines [3].

Each drug in the clinic is entered with a single barcode into the hospital drug database system. When the patient was prescribed with a medication, the information is sent electronically to the pharmacy and is entered into HIS along with his vital information. The pharmacist discloses the barcoded dose of medicine to patient's room directly. Before administrating the medicine, a barcode scanner is used to check against the patient's identification wristband barcode that one attached to patient's wristband. The BPOC system in BCMA alerts the physician when the drug cannot match with the ordered entry. The BPOC [3] system is designed to make sure that the right drug is given to the right patient via the right route in the right amount and at the right time. This information is referred to as the "five rights." The system not only reduces the medication errors but also saves the time at billing and pharmacy of an in-patient. It basically integrates the smart pump with infusion parameters based on the physician's order. It automatically documents the administration process. In this system, the manual programming of the nurses is avoided. Instead of that, the nurse is required to confirm the programming and the documentation.

\section{WIRELESS CONNECTIVITY}

The wireless connectivity can provide the direct communication of the infusion pump connected to a particular patient. Moreover, it is used to communicate the BCMA, other information, and the HIS. It is an expanded capability to BCMA infusion system means, the capability of the BCMA infusion is expanded through a wireless connectivity. The pump can be programmed remotely by wireless network.

With wireless connectivity, the data scanned for the patient via pharmacy is directly transferred to the pump. This triggers the process of IV bag infusion. As discussed, the infusion pumps are computerized pumps designed for continuous delivery of high alert drugs.

\section{DERS}

The introduction of DERS into the pumps improves the safety of infusion therapy via IV [2].

- $\quad$ Errors occurring with pump programming have been identified by this system.

- The DERS logs the patient context, and it is used to analyze the specific Events to alert the high-risk events and incorrectly programmed infusion.

The DERS of infusion pumps is a very big drug library with radio connectivity to add new drug libraries and pull some log information from the pump. The Log- analysis software is to access the clinical practical tracks. It includes the data of dosage rates and warnings against these data. This log can be used by the clinicians for later purpose and are even useful when adding new drug library and to perform some proactive risk assessments for high-risk drug deliveries. This Integration system need to interact with pump server to send and update their soft wares.

\section{Integration of devices}

The medicines used during narrow therapeutic index are very high alert medicines. Hence, careful monitoring should be necessary to ensure any abnormalities should be happen. As continuous manual monitoring is not possible for nurses, one nurse can be assigned with some other works. To fulfill, the vital parameters need to be monitored. As the pump cannot monitor these values, vital monitoring parameters devices should be integrated with PCA pump. For example, the integration of PCA pump with pulse oximetry and capnography along with automatic pause protocol (with alarm) alerts the pump if abnormalities happen, i.e., if oxygen level and/or respiratory rate reduced to dangerous levels, an added safety for PCA pump can cares

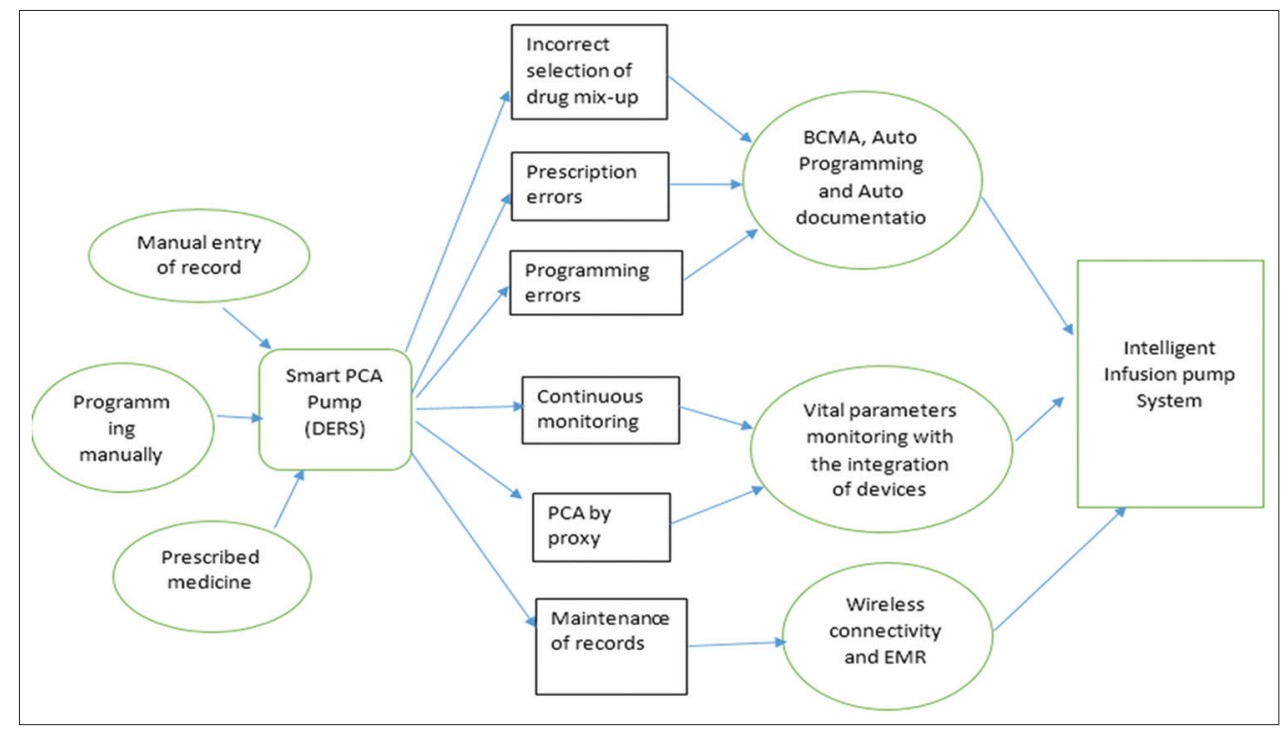

Fig. 1: Smart infusion pump errors and proposed solutions 


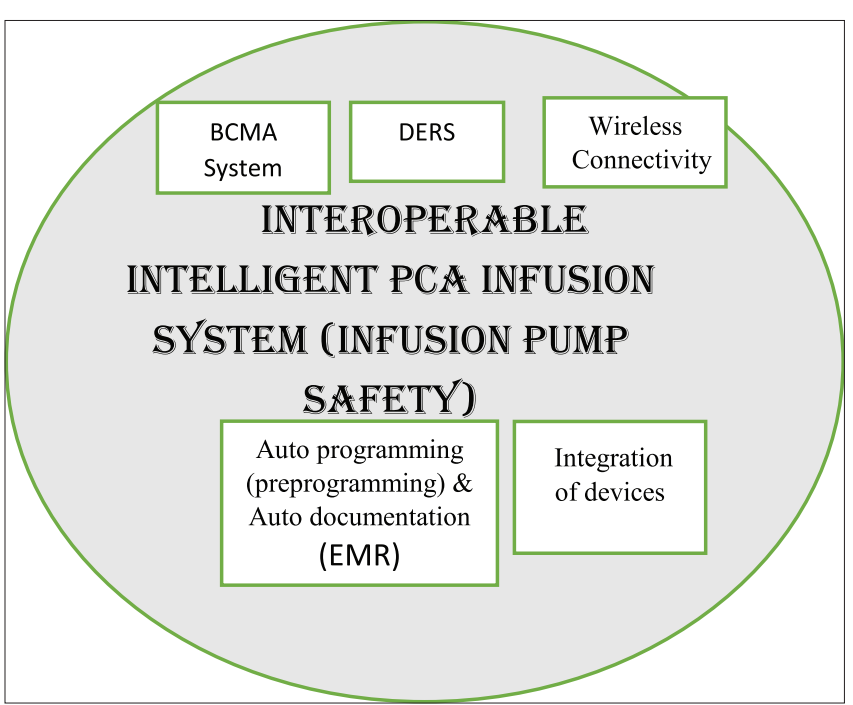

Fig. 2: Interoperable intelligent infusion system

the patients in medical/surgical units. Hence, the interoperability technology need to be used to communicate with two separate devices and systems to perform similar monitoring of a patient in response to infusion of drug.

\section{Auto programming and auto documentation}

- This process will occur when BCMA is integrated with the HIS along with the wireless connectivity. According to the BCMA, a patient is maintained with unique ID called as barcode. Moreover, this ID is generated by one of the barcoded software in the BCMA system. Using this ID, all the patients' history, prescription, and medicines are connected into the HIS.

- The prescribed medicine ordered by the physician should be entered into EMR using the CPOE. Then, the druggist uses this EMR to ensure the proper medicine to one particular patient. This avoids the prescription errors of PCA pump. Once the medicine reaches to the patient, a nurse at the care unit scans the patient wristband and drug label to verify the right patient, medication, time, dose, and route against the order in the EMR. This process is known as Auto documentation.

- Before infusion, the patient armband is scan again and that of the barcode on the smart pump. This process is used to set the ordered infusion parameters from the EMR [3]. This initiates the pump with infusion program. Then, the nurse validates the infusion information, agrees the data, and then she starts the infusion process. This automatic setting of the infusion parameters of the pump is termed as auto programming.

- While the infusion process is happening, the DERS software in the pump checks the safety features by protecting the pump against errors in manual programming. The initial settings and all subsequent changes, the rate, dose, infused volume of medication, and other data are wirelessly [3] sent back to the patient's EMR from the smart pump for the nurse to review and accept into the EMR. This avoids the manual entry of the medical records.

- Auto-documentation makes the nursing procedures very simpler by keeping everything into recorded form. This will be more useful when the patient is connected to multiple infusion pumps [3]. When multiple infusions are connected, it is so much delayed to access the information and even introduces errors. With this auto documentation, collection of data is very easy and the nursing tasks are reducing a lot and it makes the nurse to focus more on patient care.

- The automation in programming and documentation reduces the manual programming, errors. Moreover, the task of the doctor is just to confirm the infusion auto programming and its parameters.

\section{CONCLUSION}

Normally, this infusion pump is used for Intensive care patients. An interoperable interface is required to improve the patient safety for PCA infusion monitoring [9]. The integration of wireless connectivity of HIS to BCMA can decline the medication errors by $12 \%$ [3]. However, this still needs some improvement to make use of the infusion pumps for household purposes. This technology automates the physician's prescription order, infusion parameters into pump directly. This technology automates the physician's prescription order, infusion parameters into pump directly and all subsequent changes into the EMR automatically. Hence, in this, the clinician has to just confirm the pump programming and infusion record [6]. Here, the solution is considered with respect to the PCA infusion pump and the work can be progressed to meet the requirements of infusion system for ambient life scenarios.

\section{REFERENCES}

1. Goldman JM. Medical Director, Partners HealthCare Biomedical Engineering Director, Integraing the Clinical Environment to Improve Patient Safety at the Sharp edge of healthcare Delivery, CIMIT/ MGH Program on Medical Device Plug--and---Play Interoperability (MD PnP) AGending Anesthesiologist, MassachuseGs General Hospital, MIT SDM 2011 Conference on Systems Thinking forContemporary-Challenges. Available from: https://www.sdm.mit. edu/systemsthinkingconference/2011/docs/Goldman_2011-SystemsThinking-Conference.pdf.

2. Ritter HT III ${ }^{\mathrm{rd}}$. Making patient-controlled analgesia safer for patients. Pa Patient Saf Advis 2011;8(3):94-9.

3. Vanderveen T. From Smart Pumps to Intelligent Infusion Systems The Promise of Interoperability, Published on Tuesday, 27 May 2014, Patient Safety and Quality Health Care. Available from: http://www. psqh.com/analysis/from-smart-pumps-to-intelligent-infusion-systemsthe-promise-of-interoperability.

4. Leonard P, Beattie TF, Addison PS, Watson JN. Standard pulse oximeters can be used to monitor respiratory rate. Emerg Med J 2003;20(6):524-5.

5. Bench. Tech Nation Staffon posted in ECRI: In depth - Dose error reduction systems. In: ECRI Updates, Magazine. Ludington, MI: The Bench; 2014.

6. Cummings K, McGowan R. Smart infusion pumps are selectivelyintelligent. Nursing 2011;41(3):58-9.

7. Koutnik E. Infusion Pumps are Reducing Medication Errors. Available from: http://www.pharmacytimes.com/print.php.

8. Reston J. Making Health Care Safer II: An Updated Critical Analysis of the Evidence for Patient Safety Practices, Chapter 6 Smart Pumps and Other Protocols for Infusion Pumps: Brief Review.

9. Rani SU, Narayanan VN. Interoperable framework solution to ICU health care monitoring. ICTACT J Commun Technol 2015;6(1):1072-9.

10. Available from: http://www.promisetoamanda.org/2012/06/07/hai.

11. Available from: http://www.prnewswire.com/news-releases/deathof-18-year-old-amanda-abbiehl-and-opioid-induced-respiratorydepression-physician-patient-alliance-for-health--safety-releasespodcast-on-fifth-death-anniversary-300115196.html.

12. Available from: http://www.searchhealthit.techtarget.com/definition/ Bar-Coded-Medication-administration.

13. Institute for Safe Medication Practices. April, 1; 2009. Available from: https://www.ismp.org/newsletters/acutecare/archives.

14. Adverse Events Involving Intravenous Patient Controlled Analgesia; 2007. Available from: https://www.ncbi.nlm.nih.gov/ pubmed/17617499. 